\title{
Co-evolution of Language and of the Language Acquisition Device
}

\author{
Ted Briscoe \\ ejb@cl.cam.ac.uk \\ Computer Laboratory \\ University of Cambridge \\ Pembroke Street \\ Cambridge CB2 3QG, UK
}

\begin{abstract}
A new account of parameter setting during grammatical acquisition is presented in terms of Generalized Categorial Grammar embedded in a default inheritance hierarchy, providing a natural partial ordering on the setting of parameters. Experiments show that several experimentally effective learners can be defined in this framework. Evolutionary simulations suggest that a learner with default initial settings for parameters will emerge, provided that learning is memory limited and the environment of linguistic adaptation contains an appropriate language.
\end{abstract}

\section{Theoretical Background}

Grammatical acquisition proceeds on the basis of a partial genotypic specification of (universal) grammas (UG) complemented with a learning procedure enabling the child to complete this specification appropriately. The parameter setting framework of Chomsky (1981) claims that learning involves fixing the values of a finite set of finite-valued parameters to select a single fully-specified grammar from within the space defined by the genotypic specification of UG. Formal accounts of parameter setting have been developed for small fragments but even in these search spaces contain local maxima and subset-superset relations which may cause a learner to converge to an incorrect grammar (Clark, 1992; Gibson and Wexler, 1994; Niyogi and Berwick, 1995). The solution to these problems involves defining dofault, unnarked initial values for (some) paramcters and/or ordering the setting of parameters during learning.

Bickorton (1984) argues for the Bioprogram Hypothesis as an explanation for universal similarities between historically unrelated creoles, and for the rapid increase in grammatical complexity accompanying the transition from pidgin to creole languages. From the perspective of the parameters framework, the Bioprogram Hypothesis claims that children are endowed genetically with a UG which, by default, specifies the stereotypical core creole grammar, with right-branching syntax and subject-verb-object order, as in Saramaccan. Others working within the parameters framework have proposed unmarked, default parameters (e.g. Lightfoot, 1991), but the Bioprogram Hypothesis can be interpreted as towards one end of a continuum of proposals ranging from all parameters initially unset to all set to default values.

\section{The Language Acquisition Device}

A model of the Language Acquisition Device (LAD) incorporates a UG with associated parameters, a parser, and an algorithm for updating initial parameter settings on parse failure during learning.

\subsection{The Grammar (set)}

Basic categorial grammar (CG) uses one rule of application which combines a functor category (containing a slash) with an argument category to form a derived category (with one less slashed argument category). Grammatical constraints of order and agreement are captured by only allowing directed application to adjacent matching categories. Generalized Categorial Grammar (GCG) extends CG with further rule schemata. ${ }^{1}$ The rules of FA, BA, generalized weak permutation (P) and backward and forward composition $(\mathrm{FC}, \mathrm{BC})$ are given in Figure 1 (where $\mathrm{X}, \mathrm{Y}$ and $\mathrm{Z}$ are category variables, $\mid$ is a variable over slash and backslash, and ... denotes zero or more further functor arguments). Once permutation is included, several semantically

\footnotetext{
${ }^{1}$ Wood $(1993)$ is a general introduction to Categorial Grammar and extensions to the basic theory. The most closely related theories to that presented here are those of Steedman (e.g. 1988) and Hoffman (1995).
} 


\begin{tabular}{|c|c|}
\hline \multicolumn{2}{|r|}{ Forward Application: } \\
\hline$X / Y Y \Rightarrow X$ & $\lambda \mathrm{y}[\mathrm{X}(\mathrm{y})](\mathrm{y}) \Rightarrow \mathrm{X}(\mathrm{y})$ \\
\hline \multicolumn{2}{|r|}{ Backward Application: } \\
\hline$Y X \backslash Y \Rightarrow X$ & $\lambda \mathrm{y}[\mathrm{X}(\mathrm{y})](\mathrm{y}) \Rightarrow \mathrm{X}(\mathrm{y})$ \\
\hline \multicolumn{2}{|r|}{ Forward Composition: } \\
\hline $\mathrm{X} / \mathrm{Y} \mathrm{Y} / \mathrm{Z} \Rightarrow \mathrm{X} / \mathrm{Z}$ & $\lambda \mathrm{y}[\mathrm{X}(\mathrm{y})] \lambda \mathrm{z}[\mathrm{Y}(\mathrm{z})] \Rightarrow \lambda \mathrm{z}[\mathrm{X}(\mathrm{Y}(\mathrm{z}))]$ \\
\hline \multicolumn{2}{|r|}{ Backward Composition: } \\
\hline$Y \backslash Z X \backslash Y \Rightarrow X \backslash Z$ & $\lambda \mathrm{z}[\mathrm{Y}(\mathrm{z})] \lambda \mathrm{y}[\mathrm{X}(\mathrm{y})] \Rightarrow \lambda \mathrm{z}[\mathrm{X}(\mathrm{Y}(\mathrm{z}))]$ \\
\hline \multicolumn{2}{|c|}{ (Generalized Weak) Permutation: } \\
\hline$\left(\mathrm{X} \mid \mathrm{Y}_{1}\right) \ldots \mid \mathrm{Y}_{n} \Rightarrow$ & $\lambda \mathrm{y}_{n} \ldots, \mathrm{y}_{1}\left[\mathrm{X}\left(\mathrm{y}_{1} \ldots, \mathrm{y}_{n}\right)\right] \Rightarrow \lambda \mathrm{y}_{1}, \mathrm{y}_{n} \ldots\left[\mathrm{X}\left(\mathrm{y}_{1} \ldots, \mathrm{y}_{n}\right)\right]$ \\
\hline
\end{tabular}

Figure 1: GCG Rule Schemata

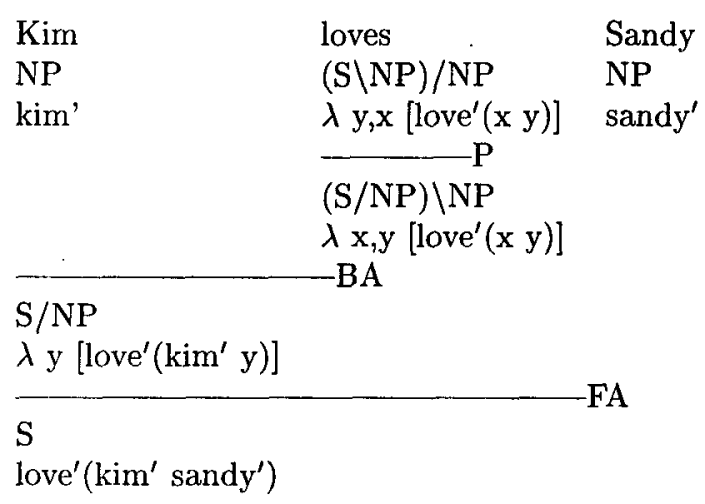

Figure 2: GCG Derivation for Kim loves Sandy

equivalent derivations for Kim loves Sandy become available, Figure 2 shows the non-conventional leftbranching one. Composition also allows alternative non-conventional semantically equivalent (leftbranching) derivations.

GCG as presented is inadequate as an account of UG or of any individual grammar. In particular, the definition of atomic categories needs extending to deal with featural variation (e.g. Bouma and van Noord, 1994), and the rule schemata, especially composition and weak permutation, must be restricted in various parametric ways so that overgeneration is prevented for specific languages. Nevertheless, GCG does represent a plausible kernel of UG; Hoffman $(1995,1996)$ explores the descriptive power of a very similar system, in which generalized weak permutation is not required because functor arguments are interpreted as multisets. She demonstrates that this system can handle (long-distance) scrambling elegantly and generates mildly context-sensitive languages (Joshi et al, 1991).

The relationship between GCG as a theory of UG (GCUG) and as a the specification of a particular grammar is captured by embedding the theory in a default inheritance hierarchy. This is represented as a lattice of typed default feature structures (TDFSs) representing subsumption and default inheritance relationships (Lascarides et al, 1996; Lascarides and Copestake, 1996). The lattice defines intensionally the set of possible categories and rule schemata via type declarations on nodes. For example, an intransitive verb might be treated as a subtype of verb, inheriting subject directionality by default from a type gendir (for general direction). For English, gendir is default right but the node of the (intransitive) functor category, where the directionality of subject arguments is specified, overrides this to left, reflecting the fact that English is predominantly right-branching, though subjects appear to the left of the verb. A transitive verb would inherit structure from the type for intransitive verbs and an extra NP argument with default directionality specified by gendir, and so forth. ${ }^{2}$

For the purposes of the evolutionary simulation described in $\S 3, \mathrm{GC}(\mathrm{U}) \mathrm{Gs}$ are represented as a sequence of $p$-settings (where $p$ denotes principles or parameters) based on a flat (ternary) sequential encoding of such default inheritance lattices. The in-

\footnotetext{
${ }^{2}$ Bouma and van Noord (1994) and others demonstrate that CGs can be embedded in a constraint-based representation. Briscoe (1997a,b) gives further details of the encoding of GCG in TDFSs.
} 


\begin{tabular}{|llllll|}
\hline NP & N & S & gen-dir & subj-dir & applic \\
A T & A T & A T & D R & D L & D T \\
\hline NP & gendir & applic & S & N & subj-dir \\
A T & D R & D T & A T & A T & D L \\
\hline applic & NP & N & gen-dir & subj-dir & S \\
D T & A T & A T & D R & D L & A T \\
\hline$\ldots$ & & & & & \\
\hline
\end{tabular}

Figure 3: Sequential encodings of the grammar fragment

heritance hierarchy provides a partial ordering on parameters, which is exploited in the learning procedure. For example, the atomic categories, $\mathbf{N}$, NP and $\mathbf{S}$ are each represented by a parameter encoding the presence/absence or lack of specification $(T / F / ?)$ of the category in the (U)G. Since they will be unordered in the lattice their ordering in the sequential coding is arbitrary. However, the ordering of the directional types gendir and subjdir (with values $\mathrm{L} / \mathrm{R}$ ) is significant as the latter is a more specific type. The distinctions between absolute, default or unset specifications also form part of the encoding ( $A / D / ?)$. Figure 3 shows several equivalent and equally correct sequential encodings of the fragment of the English type system outlined above.

A set of grammars based on typological distinctions defined by basic constituent order (e.g. Greenberg, 1966; Hawkins, 1994) was constructed as a (partial) GCUG with independently varying binaryvalued parameters. The eight basic language families are defined in terms of the unmarked order of verb $(\mathrm{V})$, subject $(\mathrm{S})$ and objects $(\mathrm{O})$ in clauses. Languages within families further specify the order of modifiers and specifiers in phrases, the order of adpositions and further phrasal-level ordering parameters. Figure 4 list the language-specific ordering parameters used to define the full set of grammars in (partial) order of generality, and gives examples of settings based on familiar languages such as "English", "German" and "Japanese". ${ }^{3}$ "English" defines an SVO language, with prepositions in which specifiers, complementizers and some modifiers precede heads of phrases. There are other grammars in the SVO family in which all modifers follow heads, there are postpositions, and so forth. Not all combinations of parameter settings correspond to attested languages and one entire language family (OVS) is unattested. "Japanese" is an SOV language with

\footnotetext{
${ }^{3}$ Throughout double quotes around language names are used as convenient mnemonics for familiar combinations of parameters. Since not all aspects of these actual languages are represented in the grammars, conclusions about actual languages must be made with care.
}

postpositions in which specifiers and modifiers follow heads. There are other languages in the SOV family with less consistent left-branching syntax in which specifiers and/or modifiers precede phrasal heads, some of which are attested. "German" is a more complex SOV language in which the parameter verbsecond (v2) ensures that the surface order in main clauses is usually SVO. ${ }^{4}$

There are $20 \mathrm{p}$-settings which determine the rule schemata available, the atomic category set, and so forth. In all, this CGUG defines just under 300 grammars. Not all of the resulting languages are (stringset) distinct and some are proper subsets of other languages. "English" without the rule of permutation results in a stringset-identical language, but the grammar assigns different derivations to some strings, though the associated logical forms are identical. "English" without composition results in a subset language. Some combinations of p-settings result in 'impossible' grammars (or UGs). Others yield equivalent grammars, for example, different combinations of default settings (for types and their subtypes) can define an identical category set.

The grammars defined generate (usually infinite) stringsets of lexical syntactic categories. These strings are sentence types since each is equivalent to a finite set of grammatical sentences formed by selecting a lexical instance of each lexical category. Languages are represented as a finite subset of sentence types generated by the associated grammar. These represent a sample of degree-1 learning triggers for the language (e.g. Lightfoot, 1991). Subset languages are represented by 3-9 sentence types and 'full' languages by 12 sentence types. The constructions exemplified by each sentence type and their length are equivalent across all the languages defined by the grammar set, but the sequences of lexical categories can differ. For example, two SOV language renditions of The man who Bill likes gave Fred a

\footnotetext{
${ }^{4}$ Representation of the $\mathrm{v} 1 / \mathrm{v} 2$ parameter(s) in terms of a type constraint determining allowable functor categories is discussed in more detail in Briscoe (1997b).
} 


\begin{tabular}{|l|lllllllllll|}
\hline & gen & v1 & n & subj & obj & v2 & mod & spec & relcl & adpos & compl \\
\hline Engl & R & F & R & L & R & F & R & R & R & R & R \\
Ger & R & F & R & L & L & T & R & R & R & R & R \\
Jap & L & F & L & L & L & F & L & L & L & L & $?$ \\
\hline
\end{tabular}

Figure 4: The Grammar Set - Ordering Parameters

present, one with premodifying and the other postmodifying relative clauses, both with a relative pronoun at the right boundary of the relative clause, are shown below with the differing category highlighted.

Bill likes who the-man a-present Fred gave

$\begin{array}{lllll}\mathrm{NP}_{s} & \left(\mathrm{~S} \backslash \mathrm{NP}_{s}\right) \backslash \mathrm{NP}_{o} & \mathrm{R} c \backslash\left(\mathrm{S} \backslash \mathrm{NP}_{o}\right) & \mathrm{NP}_{s} \backslash \mathbf{R c} & \mathrm{NP}_{o 2}\end{array}$ $\mathrm{NP}_{o 1}\left(\left(\mathrm{~S} \backslash \mathrm{NP}_{s}\right) \backslash \mathrm{NP}_{o 2}\right) \backslash \mathrm{NP}_{o 1}$

The-man Bill likes who a-present Fred gave

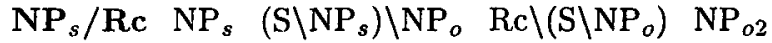
$\mathrm{NP}_{o 1}\left(\left(\mathrm{~S} \backslash \mathrm{NP}_{s}\right) \backslash \mathrm{NP}_{o 2}\right) \backslash \mathrm{NP}_{o 1}$

\subsection{The Parser}

The parser is a deterministic, bounded-context stack-based shift-reduce algorithm. The parser operates on two data structures, an input buffer or queue, and a stack or push down store. The algorithm for the parser working with a GCG which includes application, composition and permutation is given in Figure 5. This algorithm finds the most leftbranching derivation for a sentence type because Reduce is ordered before Shift. The category sequences representing the sentence types in the data for the entire language set are designed to be unambiguous relative to this 'greedy', deterministic algorithm, so it will always assign the appropriate logical form to each sentence type. However, there are frequently alternative less left-branching derivations of the same logical form.

The parser is augmented with an algorithm which computes working memory load during an analysis (e.g. Baddeley, 1992). Limitations of working memory are modelled in the parser by associating a cost with each stack cell occupied during each step of a derivation, and recency and depth of processing effects are modelled by resetting this cost each time a reduction occurs: the working memory load (WML) algorithm is given in Figure 6. Figure 7 gives the right-branching derivation for Kim loves Sandy, found by the parser utilising a grammar without permutation. The WML at each step is shown for this derivation. The overall WML (16) is higher than for the left-branching derivation (9).

The WML algorithm ranks sentence types, and
1. The Reduce Step: if the top 2 cells of the stack are occupied, then try
a) Application, if match, then apply and goto
1), else b),
b) Combination, if match then apply and goto 1), else c),
c) Permutation, if match then apply and goto 1), else goto 2)

2. The Shift Step: if the first cell of the Input Buffer is occupied, then pop it and move it onto the Stack together with its associated lexical syntactic category and goto 1 ),

else goto 3 )

3. The Halt Step: if only the top cell of the Stack is occupied by a constituent of category $\mathrm{S}$, then return Success, else return Fail

The Match and Apply operation: if a binary rule schema matches the categories of the top 2 cells of the Stack, then they are popped from the Stack and the new category formed by applying the rule schema is pushed onto the Stack.

The Permutation operation: each time step 1c) is visited during the Reduce step, permutation is applied to one of the categories in the top 2 cells of the Stack until all possible permutations of the 2 categories have been tried using the binary rules. The number of possible permutation operations is finite and bounded by the maximum number of arguments of any functor category in the grammar.

Figure 5: The Parsing Algorithm 


\begin{tabular}{|c|c|c|c|c|}
\hline Stack & Input Buffer & Operation & Step & WML \\
\hline & Kim loves Sandy & & 0 & 0 \\
\hline Kim:NP:kim' & loves Sandy & Shift & 1 & 1 \\
\hline 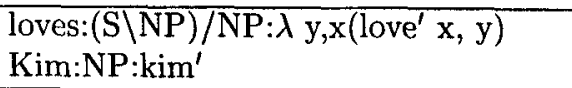 & Sandy & Shift & 2 & 3 \\
\hline $\begin{array}{l}\text { Sandy:NP:sandy' } \\
\text { loves:(S\NP)/NP: } \lambda \text { y,x(love' } x, y) \\
\text { Kim:NP:kim' }\end{array}$ & & Shift & 3 & 6 \\
\hline $\begin{array}{l}\text { loves Sandy:S/NP: } \lambda \mathrm{x}\left(\text { love }^{\prime} \mathrm{x}, \text { sandy }^{\prime}\right) \\
\text { Kim:NP:kim }\end{array}$ & & Reduce (A) & 4 & 5 \\
\hline Kim loves Sandy:S:(love ${ }^{\prime}$ kim $^{\prime}$, sandy $\left.^{\prime}\right)$ & & Reduce (A) & 5 & $\overline{1}$ \\
\hline
\end{tabular}

Figure 7: WML for Kim loves Sandy

After each parse step (Shift, Reduce, Halt (see Fig 5):

1. Assign any new Stack entry in the top cell (introduced by Shift or Reduce) a WML value of 0

2. Increment every Stack cell's WML value by 1

3. Push the sum of the WML values of each Stack cell onto the WML-record

When the parser halts, return the sum of the WMLrecord gives the total WML for a derivation

Figure 6: The WML Algorithm

thus indirectly languages, by parsing each sentence type from the exemplifying data with the associated grammar and then taking the mean of the WML obtained for these sentence types. "English" with Permutation has a lower mean WML than "English" without Permutation, though they are stringset-identical, whilst a hypothetical mixture of "Japanese" SOV clausal order with "English" phrasal syntax has a mean WML which is $25 \%$ worse than that for "English". The WML algorithm is in accord with existing (psycholinguisticallymotivated) theories of parsing complexity (e.g. Gibson, 1991; Hawkins, 1994; Rambow and Joshi, 1994).

\subsection{The Parameter Setting Algorithm}

The parameter setting algorithm is an extension of Gibson and Wexler's (1994) Trigger Learning Algorithm (TLA) to take account of the inheritancebased partial ordering and the role of memory in learning. The TLA is error-driven - parameter settings are altered in constrained ways when a learner cannot parse trigger input. Trigger input is defined as primary linguistic data which, because of its structure or context of use, is determinately unparsable with the correct interpretation (e.g. Lightfoot, 1991). In this model, the issue of ambiguity and triggers does not arise because all sentence types are treated as triggers represented by p-setting schemata. The TLA is memoryless in the sense that a history of parameter (re)settings is not maintained, in principle, allowing the learner to revisit previous hypotheses. This is what allows Niyogi and Berwick (1995) to formalize parameter setting as a Markov process. However, as Brent (1996) argues, the psychological plausibility of this algorithm is doubtful - there is no evidence that children (randomly) move between neighbouring grammars along paths that revisit previous hypotheses. Therefore, each parameter can only be reset once during the learning process. Each step for a learner can be defined in terms of three functions: P-SETTING, GRAMMAR and PARSER, as:

$$
\operatorname{PARSER}_{i}\left(\text { GRAMMAR }_{i}\left(\text { P-SETTING }_{i}\left(\text { Sentence }_{j}\right)\right)\right)
$$

A p-setting defines a grammar which in turn defines a parser (where the subscripts indicate the.output of each function given the previous trigger). A parameter is updated on parse failure and, if this results in a parse, the new setting is retained. The algorithm is summarized in Figure 8. Working memory grows through childhood (e.g. Baddeley, 1992), and this may assist learning by ensuring that trigger sentences gradually increase in complexity through the acquisition period (e.g. Elman, 1993) by forcing the learner to ignore more complex potential triggers that occur early in the learning process. The WML of a sentence type can be used to determine whether it can function as a trigger at a particular stage in learning. 


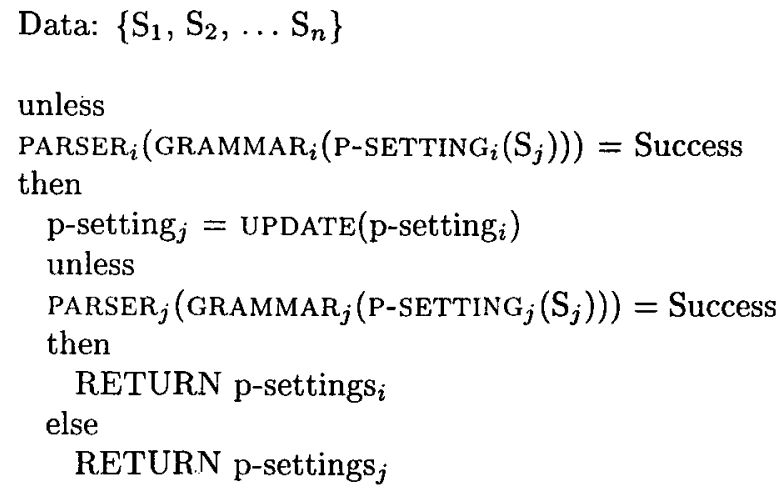

Update:

Reset the first (most general) default or unset parameter in a left-to-right search of the p-set according to the following table:

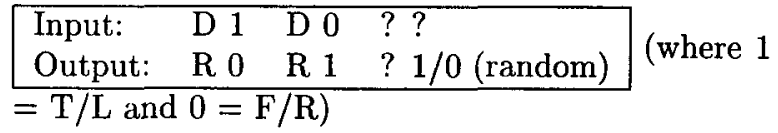

Figure 8: The Learning Algorithm

\section{The Simulation Model}

The computational simulation supports the evolution of a population of Language Agents (LAgts), similar to Holland's (1993) Echo agents. LAgts generate and parse sentences compatible with their current p-setting. They participate in linguistic interactions which are successful if their p-settings are compatible. The relative fitness of a LAgt is a function of the proportion of its linguistic interactions which have been successful, the expressivity of the language(s) spoken, and, optionally, of the mean WML for parsing during a cycle of interactions. An interaction cycle consists of a prespecified number of individual random interactions between LAgts, with generating and parsing agents also selected randomly. LAgts which have a history of mutually successful interaction and high fitness can 'reproduce'. A LAgt can 'live' for up to ten interaction cycles, but may 'die' earlier if its fitness is relatively low. It is possible for a population to become extinct (for example, if all the initial LAgts go through ten interaction cycles without any successful interaction occurring), and successful populations tend to grow at a modest rate (to ensure a reasonable proportion of adult speakers is always present). LAgts learn during a critical period from ages 1-3 and reproduce from 4-10, parsing and/or generating any language learnt throughout their life.

During learning a LAgt can reset genuine param-
Variables

Population Size

Interaction Cycle

Simulation Run

Crossover Probability

Mutation Probability

Learning

Typical Values

2K Interactions

50 Cycles

0

memory limited yes

critical period yes

Figure 9: The Simulation Options

(Cost/Benefits per sentence (1-6); summed for each LAgt at end of an interaction cycle and used to calculate fitness functions (7-8)):

1. Generate cost: 1 (GC)

2. Parse cost: 1 (PC)

3. Generate subset language cost: 1 (GSC)

4. Parse failure cost: 1 (PF)

5. Parse memory cost: WML(st)

6. Interaction success benefit: 1 (SI)

7. Fitness(WML): $\frac{S I}{G C+P C} \times \frac{G C}{G C+G S C} \times \frac{1}{\left(\frac{W M L}{P C-P F}\right)}$

8. Fitness $(\neg \mathrm{WML}): \frac{S I}{G C+P C} \times \frac{G C}{G C+G S C}$

Figure 10: Fitness Functions

eters which either were unset or had default settings 'at birth'. However, p-settings with an absolute value (principles) cannot be altered during the lifetime of an LAgt. Successful LAgts reproduce at the end of interaction cycles by one-point crossover of (and, optionally, single point mutation of) their initial p-settings, ensuring neo-Darwinian rather than Lamarckian inheritance. The encoding of p-settings allows the deterministic recovery of the initial setting. Fitness-based reproduction ensures that successful and somewhat compatible p-settings are preserved in the population and randomly sampled in the search for better versions of universal grammar, including better initial settings of genuine parameters. Thus, although the learning algorithm per se is fixed, a range of alternative learning procedures can be explored based on the definition of the inital set of parameters and their initial settings. Figure 9 summarizes crucial options in the simulation giving the values used in the experiments reported in $\S 4$ and Figure 10 shows the fitness functions. 


\section{Experimental Results}

\subsection{Effectiveness of Learning Procedures}

Two learning procedures were predefined - a default learner and an unset learner. These LAgts were initialized with p-settings consistent with a minimal inherited CGUG consisting of application with NP and $\mathrm{S}$ atomic categories. All the remaining p-settings were genuine parameters for both learners. The unset learner was initialized with all unset, whilst the default learner had default settings for the parameters gendir and subjdir and argorder which specify a minimal SVO right-branching grammar, as well as default (off) settings for comp and perm which determine the availability of Composition and Permutation, respectively. The unset learner represents a 'pure' principles-and-parameters learner. The default learner is modelled on Bickerton's bioprogram learner.

Each learner was tested against an adult LAgt initialized to generate one of seven full languages in the set which are close to an attested language; namely, "English" (SVO, predominantly right-branching), "Welsh" (SVOv1, mixed order), "Malagasy" (VOS, right-branching), "Tagalog" (VSO, right-branching), "Japanese" (SOV, left-branching), "German" (SOVv2, predominantly right-branching), "Hixkaryana" (OVS, mixed order), and an unattested full OSV language with leftbranching syntax. In these tests, a single learner interacted with a single adult. After every ten interactions, in which the adult randomly generated a sentence type and the learner attempted to parse and learn from it, the state of the learner's p-settings was examined to determine whether the learner had converged on the same grammar as the adult. Table 1 shows the number of such interaction cycles (i.e. the number of input sentences to within ten) required by each type of learner to converge on each of the eight languages. These figures are each calculated from 100 trials to a $1 \%$ error rate; they suggest that, in general, the default learner is more effective than the unset learner. However, for the OVS language (OVS languages represent $1.24 \%$ of the world's languages, Tomlin, 1986), and for the unattested OSV language, the default (SVO) learner is less effective. So, there are at least two learning procedures in the space defined by the model which can converge with some presentation orders on some of the grammars in this set. Stronger conclusions require either exhaustive experimentation or theoretical analysis of the model of the type undertaken by Gibson and Wexler (1994) and Niyogi and Berwick (1995).

\begin{tabular}{|r|lll|}
\hline & Unset & Default & None \\
\hline WML & 15 & 39 & 26 \\
$\neg$ WML & 34 & 17 & 29 \\
\hline
\end{tabular}

Table 2: Overall preferences for parameter types

\subsection{Evolution of Learning Procedures}

In order to test the preference for default versus unset parameters under different conditions, the five parameters which define the difference between the two learning procedures were tracked through another series of 50 cycle runs initialized with either 16 default learning adult speakers and 16 unset learning adult speakers, with or without memory-limitations during learning and parsing, speaking one of the eight languages described above. Each condition was run ten times. In the memory limited runs, default parameters came to dominate some but not all populations. In a few runs all unset parameters disappeared altogether. In all runs with populations initialized to speak "English" (SVO) or "Malagasy" (VOS) the preference for default settings was $100 \%$. In 8 runs with "Tagalog" (VSO) the same preference emerged, in one there was a preference for unset parameters and in the other no clear preference. However, for the remaining five languages there was no strong preference.

The results for the runs without memory limitations are different, with an increased preference for unset parameters across all languages but no clear $100 \%$ preference for any individual language. Table 2 shows the pattern of preferences which emerged across 160 runs and how this was affected by the presence or absence of memory limitations.

To test whether it was memory limitations during learning or during parsing which were affecting the results, another series of runs for "English" was performed with either memory limitations during learning but not parsing enabled, or vice versa. Memory limitations during learning are creating the bulk of the preference for a default learner, though there appears to be an additive effect. In seven of the ten runs with memory limitations only in learning, a clear preference for default learners emerged. In five of the runs with memory limitations only in parsing there appeared to be a slight preference for defaults emerging. Default learners may have a fitness advantage when the number of interactions required to learn successfully is greater because they. will tend to converge faster, at least to a subset language. This will tend to increase their fitness over unset learners who do not speak any language until further into the 


\begin{tabular}{|l|llllllll|}
\hline Learner & \multicolumn{10}{|c|}{ Language } \\
\hline & SVO & SVOv1 & VOS & VSO & SOV & SOVv2 & OVS & OSV \\
\hline Unset & 60 & 80 & 70 & 80 & 70 & 70 & 70 & 70 \\
Default & 60 & 60 & 60 & 60 & 60 & 60 & 80 & 70 \\
\hline
\end{tabular}

Table 1: Effectiveness of Two Learning Procedures

learning period.

The precise linguistic environment of adaptation determines the initial values of default parameters which evolve. For example, in the runs initialized with 16 unset learning "Malagasy" VOS adults and 16 default (SVO) learning VOS adults, the learning procedure which dominated the population was a variant VOS default learner in which the value for subjdir was reversed to reflect the position of the subject in this language. In some of these runs, the entire population evolved a default subjdir 'right' setting, though some LAgts always retained unset settings for the other two ordering parameters, gendir and argo, as is illustrated in Figure 11. This suggests that if the human language faculty has evolved to be a right-branching SVO default learner, then the environment of linguistic adaptation must have contained a dominant language fully compatible with this (minimal) grammar.

\subsection{Emergence of Language and Learners}

To explore the emergence and persistence of structured language, and consequently the emergence of effective learners, (pseudo) random initialization was used. A series of simulation runs of 500 cycles were performed with random initialization of 32 LAgts' p-settings for any combination of p-setting values, with a probability of 0.25 that a setting would be an absolute principle, and 0.75 a parameter with unbiased allocation for default or unset parameters and for values of all settings. All LAgts were initialized to be age 1 with a critical period of 3 interaction cycles of 2000 random interactions for learning, a maximum age of 10 , and the ability to reproduce by crossover ( 0.9 probability) and mutation ( 0.01 probability) from 4-10. In around $5 \%$ of the runs, language(s) emerged and persisted to the end of the run.

Languages with close to optimal WML scores typically came to dominate the population quite rapidly. However, sometimes sub-optimal languages were initially selected and occasionally these persisted despite the later appearance of a more optimal language, but with few speakers. Typically, a minimal subset language dominated -- although full and intermediate languages did appear briefly, they did not survive against less expressive subset languages with a lower mean WML. Figure 12 is a typical plot of the emergence (and extinction) of languages in one of these runs. In this run, around 10 of the initial population converged on a minimal OVS language and 3 others on a VOS language. The latter is more optimal with respect to WML and both are of equal expressivity so, as expected, the VOS language acquired more speakers over the next few cycles. A few speakers also converged on VOS-N, a more expressive but higher WML extension of VSO-N-GWPCOMP. However, neither this nor the OVS language survived beyond cycle 14. Instead a VSO language emerged at cycle 10 , which has the same minimal expressivity of the VOS language but a lower WML (by virtue of placing the subject before the object) and this language dominated rapidly and eclipsed all others by cycle 40 .

In all these runs, the population settled on subset languages of low expressivity, whilst the percentage of absolute principles and default parameters increased relative to that of unset parameters (mean $\%$ change from beginning to end of runs: $+4.7,+1.5$ and -6.2 , respectively). So a second identical set of ten was undertaken, except that the initial population now contained two SOV-V2 "German" speaking unset learner LAgts. In seven of these runs, the population fixed on a full SOV-V2 language, in two on the intermediate subset language SOV-V2-N, and in one on the minimal subset language SOV-V2-NGWP-COMP. These runs suggest that if a full language defines the environment of adaptation then a population of randomly initialized LAgts is more likely to converge on a (related) full language. Thus, although the simulation does not model the development of expressivity well, it does appear that it can model the emergence of effective learning procedures for (some) full languages. The pattern of language emergence and extinction followed that of the previous series of runs: lower mean WML languages were selected from those that emerged during the run. However, often the initial optimal SVO-V2 itself was lost before enough LAgts evolved capable of learning this language. In these runs, changes in the percentages of absolute, default or unset psettings in the population show a marked difference: 


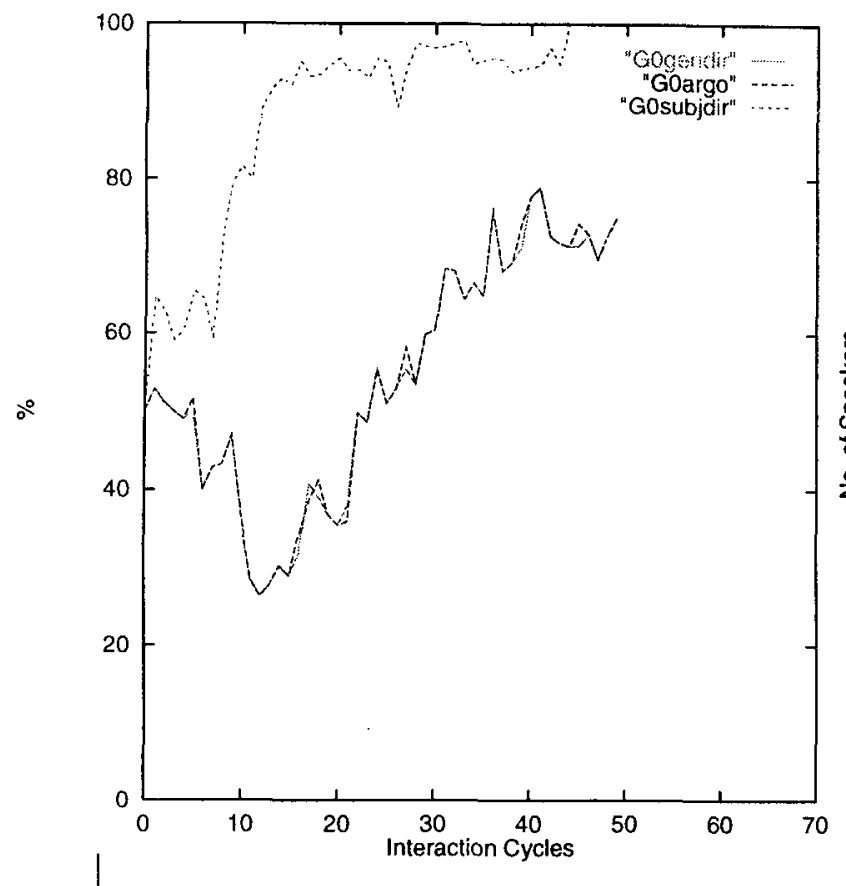

Figure 11: Percentage of each default ordering parameter

the mean number of absolute principles declined by $6.1 \%$ and unset parameters by $17.8 \%$, so the number of default parameters rose by $23.9 \%$ on average between the beginning and end of the 10 runs. This may reflect the more complex linguistic environment in which (incorrect) absolute settings are more likely to handicap, rather than simply be irrelevant to, the performance of the LAgt.

\section{Conclusions}

Partially ordering the updating of parameters can result in (experimentally) effective learners with a more complex parameter system than that studied previously. Experimental comparison of the default (SVO) learner and the unset learner suggests that the default learner is more efficient on typologically more common constituent orders. Evolutionary simulation predicts that a learner with default parameters is likely to emerge, though this is dependent both on the type of language spoken and the presence of memory limitations during learning and parsing. Moreover, a SVO bioprogram learner is only likely to evolve if the environment contains a dominant SVO language.

The evolution of a bioprogram learner is a manifestation of the Baldwin Effect (Baldwin, 1896) genetic assimilation of aspects of the linguistic envi-

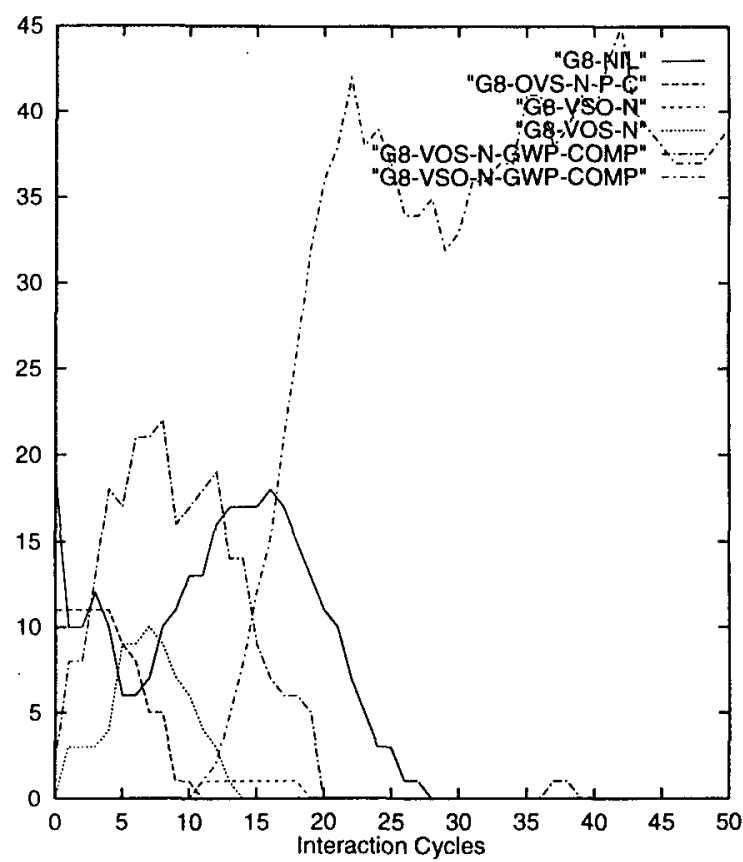

Figure 12: Emergence of language(s)

ronment during the period of evolutionary adaptation of the language learning procedure. In the case of grammar learning this is a co-evolutionary process in which languages (and their associated grammars) are also undergoing selection. The WML account of parsing complexity predicts that a right-branching SVO language would be a near optimal selection at a stage in grammatical development when complex rules of reordering such as extraposition, scrambling or mixed order strategies such as v1 and v2 had not evolved. Briscoe (1997a) reports further experiments which demonstrate language selection in the model.

Though, simulation can expose likely evolutionary pathways under varying conditions, these might have been blocked by accidental factors, such as genetic drift or bottlenecks, causing premature fixation of alleles in the genotype (roughly corresponding to certain p-setting values). The value of the simulation is to, firstly, show that a bioprogram learner could have emerged via adaptation, and secondly, to clarify experimentally the precise conditions required for its emergence. Since in many cases these conditions will include the presence of constraints (working memory limitations, expressivity, the learning algorithm etc.) which will remain causally manifest, further testing of any conclusions drawn must concentrate on demonstrating the ac- 
curacy of the assumptions made about such constraints. Briscoe (1997b) evaluates the psychological plausibility of the account of parsing and working memory.

\section{References}

Baddeley, A. (1992) 'Working Memory: the interface between memory and cognition', J. of Cognitive Neuroscience, vol.4.3, 281-288.

Baldwin, J.M. (1896) 'A new factor in evolution', American Naturalist, vol.30, 441-451.

Bickerton, D. (1984) 'The language bioprogram hypothesis', The Behavioral and Brain Sciences, vol.7.2, 173-222.

Bouma, G. and van Noord, G (1994) 'Constraintbased categorial grammar', Proceedings of the 32nd Assoc. for Computational Linguistics, Las Cruces, NM, pp. 147-154.

Brent, M. (1996) 'Advances in the computational study of language acquisition', Cognition, vol.61, $1-38$.

Briscoe, E.J. (1997a, submitted) 'Language Acquisition: the Bioprogram Hypothesis and the Baldwin Effect', Language,

Briscoe, E.J. (1997b, in prep.) Working memory and its influence on the development of human languages and the human language faculty, University of Cambridge, Computer Laboratory, m.s..

Chomsky, N. (1981) Government and Binding, Foris, Dordrecht.

Clark, R. (1992) 'The selection of syntactic knowledge', Language Acquisition, vol.2.2, 83-149.

Elman, J. (1993) 'Learning and development in neural networks: the importance of starting small', Cognition, vol.48, 71-99.

Gibson, E. (1991) A Copmutational Theory of Human Linguistic Processing: Memory Limitations and Processing Breakdown, Doctoral dissertation, Carnegie Mellon University.

Gibson, E. and Wexler, K. (1994) 'Triggers', Linguistic Inquiry, vol.25.3, 407-454.

Greenberg, J. (1966) 'Some universals of grammar with particular reference to the order of meaningful elements' in J. Greenberg (ed.), Universals of Grammar, MIT Press, Cambridge, Ma., pp. 73-113.

Hawkins, J.A. (1994) A Performance Theory of Order and Constituency, Cambridge University Press, Cambridge.

Hoffman, B. (1995) The Computational Analysis of the Syntax and Interpretation of 'Free' Word Order in Turkish, PhD dissertation, University of Pennsylvania.
Hoffman, B. (1996) 'The formal properties of synchronous CCGs', Proceedings of the ESSLLI Formal Grammar Conference, Prague.

Holland, J.H. (1993) Echoing emergence: objectives, rough definitions and speculations for echo-class models, Santa Fe Institute, Technical Report 9304-023.

Joshi, A., Vijay-Shanker, K. and Weir, D. (1991) 'The convergence of mildly context-sensitive grammar formalisms' in Sells, P., Shieber, S. and Wasow, T. (ed.), Foundational Issues in Natural Language Processing, MIT Press, pp. 31-82.

Lascarides, A., Briscoe E.J. , Copestake A.A and Asher, N. (1995) 'Order-independent and persistent default unification', Linguistics and Philosophy, vol.19.1, 1-89.

Lascarides, A. and Copestake A.A. (1996, submitted) 'Order-independent typed default unification', Computational Linguistics,

Lightfoot, D. (1991) How to Set Parameters: Arguments from language Change, MIT Press, Cambridge, Ma..

Niyogi, P. and Berwick, R.C. (1995) 'A markov language learning model for finite parameter spaces', Proceedings of the 33rd Annual Meeting of the Association for Computational Linguistics, MIT, Cambridge, Ma..

Rambow, O. and Joshi, A. (1994) 'A processing model of free word order languages' in C. Clifton, L. Frazier and K. Rayner (ed.), Perspectives on Sentence Processing, Lawrence Erlbaum, Hillsdale, N.J., pp. 267-301.

Steedman, M. (1988) 'Combinators and grammars' in R. Oehrle, E. Bach and D. Wheeler (ed.), Categorial Grammars and Natural Language Structures, Reidel, Dordrecht, pp. 417-442.

Tomlin, R. (1986) Basic Word Order: Functional Principles, Routledge, London.

Wood, M.M. (1993) Categorial-Grammars, Routledge, London. 